\title{
Development and Analysis of Novel IoT Based Resistive Soil Moisture Sensor using Arduino UNO
}

\author{
Akhilesh Kandwal $^{2}$, Amit Kumar Shakya ${ }^{1 *}$, Ayushman Ramola $^{1}$, Anurag Vidhyarthi ${ }^{2}$ \\ \{akhileshindia@outlook.com, *xlamitshakya.gate2014@iee.org, pec1901_ayushman@sliet.ac.in \\ vidyarthianurag@yahoo.co.in \} \\ Department of Electronics and Communication Engineering \\ ${ }^{1}$ Sant Longowal Institute of Engineering and Technology, Sangrur, Punjab, India \\ ${ }^{2}$ Graphic Era (Deemed to be University), Dehradun, Uttarakhand, India
}

\begin{abstract}
This paper presents the designing and construction of a resistive based soil moisture sensor using the Arduino UNO board. The low-cost sensor can be an effective device that can be used in determining and monitoring water content in the soil. The system is proposed to help farmers in agricultural applications. By knowing moisture content in the soil, farmers can determine the appropriate water needed for optimum growth of plants and improve irrigation planning thus increasing the overall crop productivity. Different soil test samples with different water content are taken for observing the variations in the values of the developed sensor. The sensor is found to meet considerable accuracy to a certain extent. The fusion of soil moisture models like the Dubois model is performed with the sensing device.
\end{abstract}

Keywords. Soil moisture, sensor, Arduino UNO, microcontroller, embedded systems, Dubois model

\section{Introduction}

The amount of water quantity contained in soil is referred to as soil moisture. Soil holds the water between the pore spaces of soil and plays a significant role in plant growth [1]. Water content in soil serves as a nutrient carrier for plant growth. Measuring moisture content is important for agricultural applications. It helps farmers in managing their irrigation systems more effectively. Soil moisture measurement also has other numerous research applications, like in agricultural science and horticulture including irrigation planning, climate research, or environmental science [1], [2]. To prevent overwatering, leaching of fertilizers, golf courses are also using soil moisture sensors to increase the efficiency of their irrigation system. There is a significant need for improving irrigation planning and rational use of water resources for assisting crop productivity. Plants can readily absorb water from the soil if the optimum water is available in the soil. By knowing the exact soil. moisture conditions of their fields, farmers are able to appropriately use water resources to grow a crop; they are also able to increase yields and the quality of the crop by improved management of soil moisture during different stages of plant growth. A number of techniques have been developed over the past few decades [2]. The Gravimetric method is the standard method of measuring moisture which involves oven drying of a known volume of soil at $105^{\circ}$ and determining the weight loss [2]. This conventional method of measuring soil moisture is a difficult, complex and time-consuming process and cannot be used for rapid repetitive measurements. Many other techniques have been proposed and are practiced which offer an 
alternate method too time-consuming gravimetric sampling [2]. For quick and rapid measurement, electronic sensors such as time domain reflectometers, capacitance, impedance, and dielectric sensors are used [3], [4], [5], [6]. However, the expensive and relatively high cost of these sensors reduces its usage amongst the farmers. H. Sharma [8] developed a soil moisture sensor for monitoring the health of chile peppers they monitored their agriculture on the basis of moisture difference using volumetric water contents in their soil. Juan D. González-True [9] developed, designed and analyzed a soil moisture sensor based on the SDI-12 communication their developed sensor is helpful to understand the agriculture pattern behavior and irrigation pattern. Zhenran [10] developed a soil moisture sensor for investigating the soil surface profile at different locations. Their soil moisture sensor gives useful information about the surface condition of the land with precise accuracy. Some soil moisture measurement techniques include Gypsum block, timedomain reflectometer, Tensiometers, gravimetric method, etc. Mostly gravimetric method is used because it is quite simple in approach and application. The measurement of the moisture content using this method is expressed by eq. 1 .

moisture content

$$
=\frac{(\text { weight of original soil sample }- \text { weight of dried soil sample })}{\text { weight of original sample }} \times 100
$$

The main advantage of this method is it is accurate and reliable with a very low degree of error but the main disadvantage it is very time consuming and a lot of human labor needs to be get involved during the process of sample collection.

Therefore, in this paper, our research work focuses on making a low-cost soil moisture sensor using the gravimetric method the main disadvantage of this process is resolved by the introduction of IoT based device in the sensor setup. This resistive based sensor offers an alternate for expensive and time-consuming methods of soil moisture measurements.

\section{Working principle}

This resistive based sensor works on the principle of how soil resistance opposes the flow of electricity between the electrodes probe [3]. These electrodes are placed into the soil which acts as a variable resistor. The resistance varies depending on the water content in the soil. Movement of ions is more in wet soil compared to the dry soil which increases its conductivity. Depending upon the resistance offered, Arduino collects the proportional voltage. Fig 1 (a) represents the basic structure of the soil moisture sensor. Here external power supply is connected with the microcontroller Arduino UNO which is further connected with LCD display and the sensing probe. The data collected with the assistance of the Arduino UNO is stored in the micro SD card connected with the device. The main advantage of this setup is the connection of the SD card so that data collected in the SD card can be easily transmitted to a computer system for further processing. 


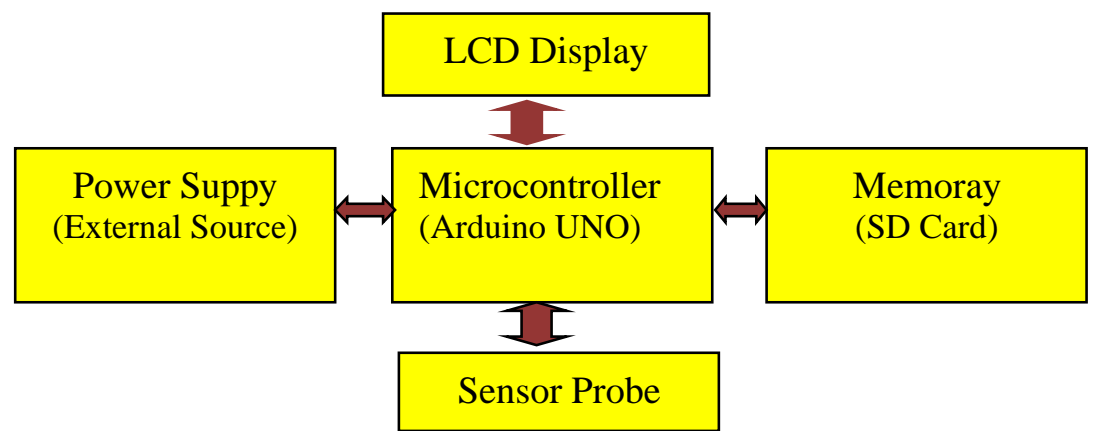

(a)

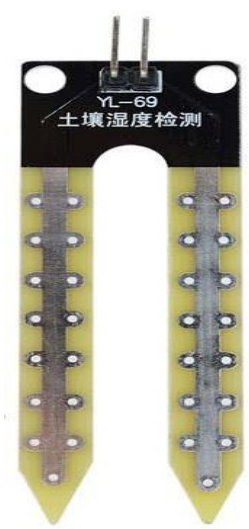

(b)

Fig. 1 (a) Block diagram of the sensor setup (b) sensing probe

The sensing probe is considered as devices that are actually responsible for the measurement of the particular property like moisture in case of the moisture sensor, the temperature in case of a temperature sensor, current in case of current sensor and so on. Thus these devices need to strong enough to tackle atmospheric distortions. In the case of soil moisture, these probes are to be inserted in the ground for moisture measurement, for soil moisture measurement the distance in which these probes need to be inserted is up to $5 \mathrm{~cm}$. Here our probe can measure moisture up to 7 $\mathrm{cm}$. The sensor setup contains a microcontroller, sensing probe, LCD display, power supply and memory source (SD card). 


\section{Construction and design procedure}

\section{A. Microcontroller}

This simple low-cost soil moisture sensor uses an Arduino UNO microcontroller board based on the Microchip AT mega 328P microcontroller. The board is equipped with an onboard serial USB port for PC (Personal computer) interface. The board also consists of analog and digital input/output (I/O) pins that are used for interfacing other circuit expansions. It was programmed with the Arduino IDE (Integrated Development Environment) using type B USB cable.

\section{B. Sensor}

The design of this sensor consists of a copper probe of a certain length which acts as a sensor. The ends of the probe are separated by a fixed distance. The probe ends are kept pointed so it can be easily inserted in the soil. The probe is kept rigid to avoid bending while inserting (Fig. 1(b)).

\section{LCD Display}

A 20×4 LCD (Liquid Crystal Display) was interfaced for displaying the sensor values. The LCD was set to display value for every ten seconds of the interval from the sensor. The output value can be directly read and displayed from an LCD display.

\section{Power Supply}

The device can be powered by an external battery source of 9 volts or a USB cable supply. It accepts voltages between 7 to 20 volts. In this system, we have used a $15000 \mathrm{mAh}$ portable power bank, which is often used for the charging of cell phones. This enables to continuously record values for even longer durations of time.

\section{E. Memory}

Apart from measuring soil moisture, monitoring moisture behavior and analyzation are also important in agricultural applications. For continuous monitoring and observation of moisture behavior of soil moisture, the Micro SD card module is interfaced. This enables the device to continuously store data readings over assigned time.

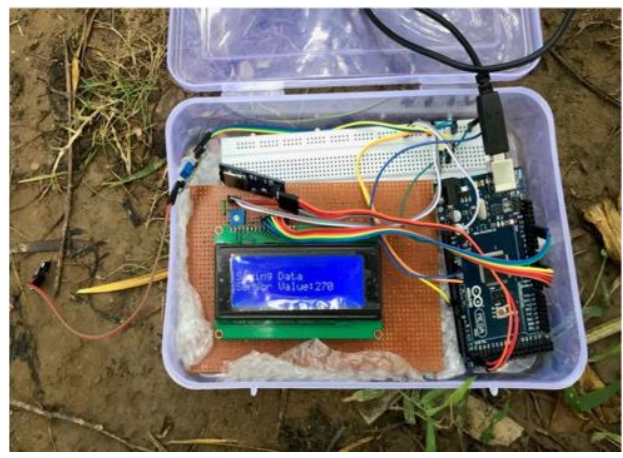

Fig. 2 Developed "SOIL MOISTURE SENSOR” setup 


\section{Experimental results}

In this test, the changes in sensor values are examined for different amounts of water content in soil samples.

a. Ten different containers were taken with the same soil sample.

b. Different amount of water content was poured in different beakers containing soil samples.

c. Samples were left for a few minutes to properly allow water to absorb and distribute in soil.

d. Further, the values were tabulated for different samples gathered from the sensor in Table I.

e. The behavioral change of sensor values with an increase in water content was analyzed using the graph plot shown in Fig. 3 (degree 1 and degree 2).

\section{A. Test 1}

TABLE I. Sensor value for different levels of water content

\begin{tabular}{|c|c|c|c|}
\hline S.No & Samples & Water added (milliliters) & Sensor Value \\
\hline 1 & 1 & 3 & 562 \\
\hline 2 & 2 & 5 & 499 \\
\hline 3 & 3 & 7 & 411 \\
\hline 4 & 4 & 10 & 382 \\
\hline 5 & 5 & 12 & 344 \\
\hline 6 & 6 & 15 & 330 \\
\hline 7 & 7 & 17 & 322 \\
\hline 8 & 8 & 20 & 314 \\
\hline 9 & 9 & 23 & 329 \\
\hline 10 & 10 & 25 & 287 \\
\hline
\end{tabular}

Polynomial fitting of degree 1 and degree 2 represents $R^{2}=0.7737$ and $R^{2}=0.8965$ respectively indicating good fitting for the sensor response. Linear fitting for degree 1 is represented by Eq. (1) and for degree 2 is represented by Eq. (2) where $x_{11}$ is the independent variable (SV) and $x_{1}$ is the dependent variable (WML).

$$
\begin{gathered}
f\left(x_{1}\right)=-0.07515\left(x_{11}\right)+42.11 \\
f\left(x_{11}\right)=0.0004227\left(x_{11}^{2}\right)-0.4339\left(x_{11}\right)+114.3
\end{gathered}
$$
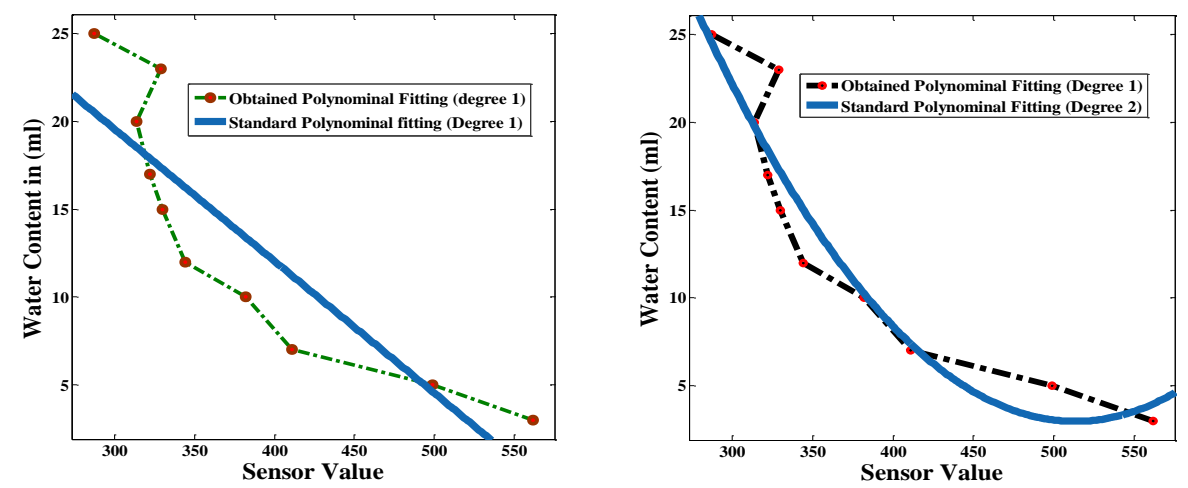
Fig.3. Polynomial fitting for the sensor response (degree1) and (degree 2)

The sensor values have been plotted for different moisture content for various samples. The result of different gathered sample values is depicted in Fig. 3. It can be seen that sensor value can be linearly correlated with soil wetness. Sensor values decrease with an increase in the wetness of the soil.

\section{B. Test 2}

For long duration and continuous monitoring of moisture behavior, the sensor was examined in the ground for a certain time.

a. The device is set and left for two hours to gather ground readings from the sensor.

b. Readings were taken on two different days with different moisture conditions.

c. Sensor values are continuously logged for every fixed 10 seconds of delay.

d. All data values are saved in Micro SD card.

TABLE II. Sensor value for a different level of water content (10 Second delay)

\begin{tabular}{|c|c|c|c|}
\hline S.No & Samples & $\begin{array}{c}\text { Water added (milliliters) } \\
\text { (WML) }\end{array}$ & $\begin{array}{c}\text { Sensor Value } \\
\text { (SV) }\end{array}$ \\
\hline 1 & 1 & 3 & 562 \\
\hline 2 & 2 & 5 & 458 \\
\hline 3 & 3 & 7 & 380 \\
\hline 4 & 4 & 10 & 314 \\
\hline 5 & 5 & 12 & 246 \\
\hline 6 & 6 & 15 & 184 \\
\hline 7 & 7 & 17 & 146 \\
\hline 8 & 8 & 20 & 108 \\
\hline 9 & 9 & 23 & 86 \\
\hline 10 & 10 & 25 & 72 \\
\hline
\end{tabular}

Polynomial fitting of degree 1 and degree 2 represents $\mathrm{R}^{2}=0.9333$ and $\mathrm{R}^{2}=0.9877$ respectively indicating good fitting for the sensor response. Linear fitting for degree 1 is represented by Eq. (3) and for degree 2 is represented by Eq. (4), where $x_{11}$ is the independent variable (SV) and $x_{1}$ is the dependent variable (WML).

$$
\begin{gathered}
f\left(x_{1}\right)=-0.04353\left(x_{11}\right)+24.83 \\
f\left(x_{1}\right)=7.722 \times 10^{-5}\left(x_{11}^{2}\right)-0.09003\left(x_{11}\right)+29.7
\end{gathered}
$$



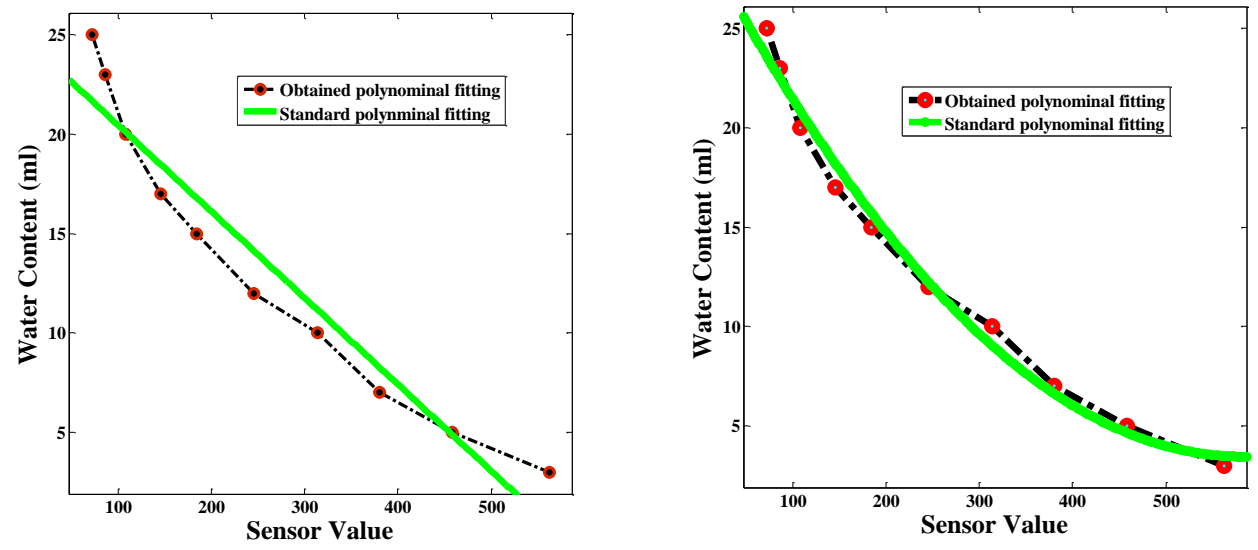

Fig.4. Polynomial fitting for the sensor response (degree1) and (degree 2)

Different changes in moisture patterns with time can be observed for different readings collected by the sensor in Fig. 3 and Fig. 4. These analyses can help in predicting the moisture behavior of the fields. Here Fig. 3 shows the normal pattern of the sensor value with no specific time gap between two different sensor values. The curve fitting of the obtained sensor value also shows the low value of r- square i.e. 0.77 and 0.89 whereas with the introduction of the time gap of $10 \mathrm{sec}$ between two soil samples the value of curve fitting $r$ - square is improved to 0.93 and 0.98 which are near to the ideal value of approx 1 . Thus it is also observed that the time gap between two soil samples is an important point to consider.

Their some standard soil moisture retrieval models like the Dubois model, Oh model, integral equation model (IEM) which are used to estimate the moisture content present in the soil specifically for the agricultural purposes under varying conditions. These models can be used to obtain the moisture conditions present in the soil. These calculate moisture content based upon polarization, incidence angle, RMS height, dielectric constant etc. The Dubois model for horizontal-horizontal (HH) and vertical-vertical (VV) polarized mode is represented by Eq. (5) and Eq.(6) respectively. Here $k$ represents wavenumber in free space, $h$ represents the RMS height, $\theta$ represents the orientation angle and $\lambda$ represents wavelength in $(\mu \mathrm{m})$.

$$
\begin{gathered}
\sigma_{H H}^{\circ}=10^{-2.75} \times \frac{\cos ^{1.5} \theta}{\sin ^{5} \theta} \times 10^{0.028 \times \in \times \tan \theta} \times(k \times h \times \sin \theta)^{1.4} \times \lambda^{0.7} \\
\sigma_{V V}^{\circ}=10^{-2.35} \times \frac{\cos ^{3} \theta}{\sin ^{3} \theta} \times 10^{0.046 \times \in \times \tan \theta} \times(k \times h \times \sin \theta)^{1.1} \times \lambda^{0.7}
\end{gathered}
$$

The HH polarization curve for $\mathrm{HH}$ scattering coefficients suggests that as the value of the dielectric constant increases $\mathrm{HH}$ polarized coefficients also increase. The numerical value of $\mathrm{HH}$ scattering coefficients also increases shown in Fig. 5 (a). The VV polarization curve also shown the same conclusion and is shown in Fig. 5 (b). The value of the dielectric constant is varied from 1 to 40 in our experiment. Our developed devices can measure soil moisture content up to $5 \mathrm{~cm}$, whereas in the experiment we have a varied height from $5 \mathrm{~cm}$ to $30 \mathrm{~cm}$ respectively. Here we have observed that polarization is directly proportional to the dielectric values. 

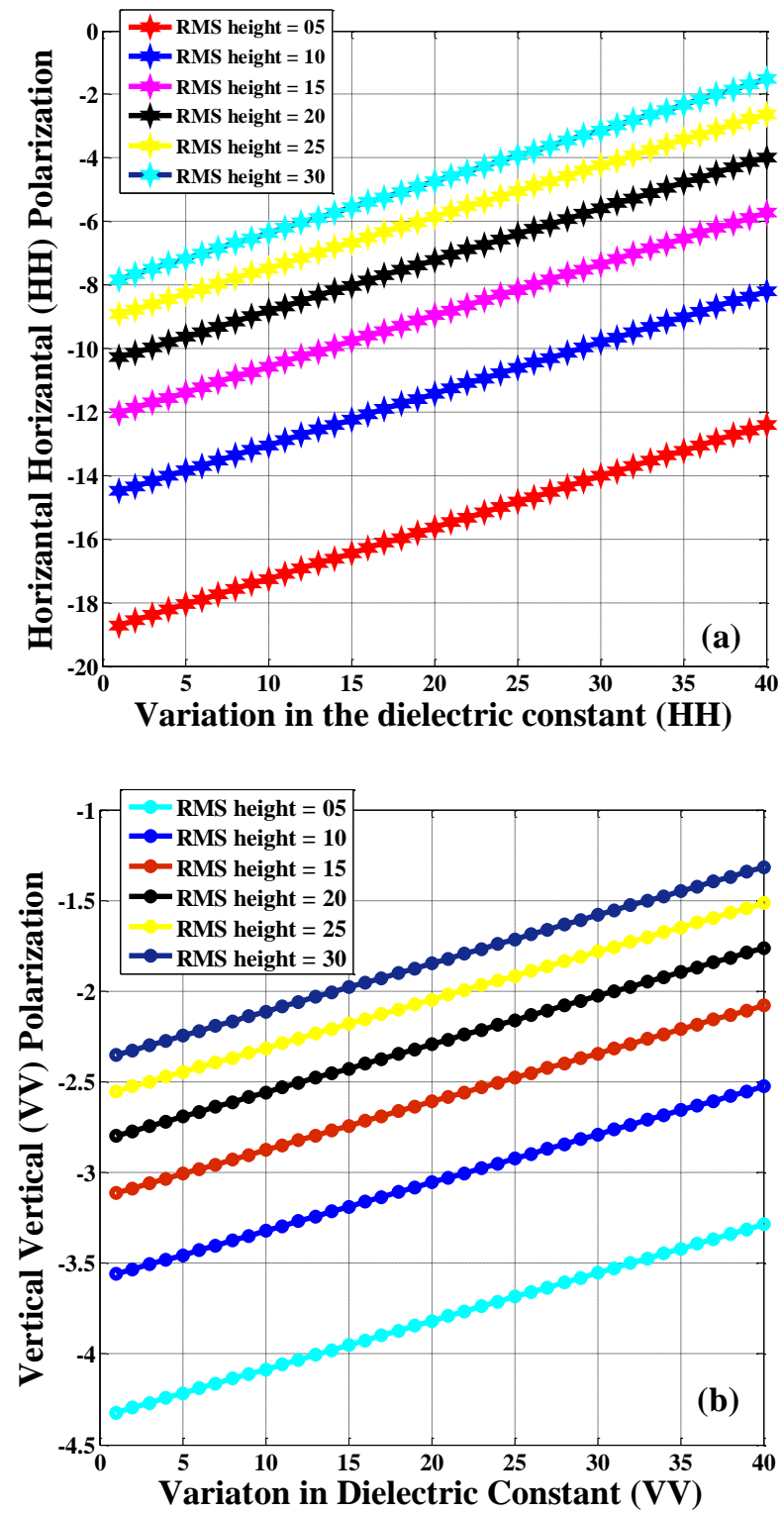

Fig.5. (a) HH polarization against dielectric constant (b) VV polarization against dielectric constant

The IoT devices can be embedded with some standard soil moisture models to provides information about various parameters to the farmers, especially for agriculture purposes. 


\section{Conclusion}

The manufacturing of the developed device is economical and can be easily affordable by farmers. By connecting a soil moisture sensor with IoT (Internet of Things) and smart irrigation systems can prevent irrigation cycles when the soil is already wet. Timely information about the moisture content can help farmers in controlling and managing the irrigation systems. Future work also involves validating and improving the calibration of the device so that it can be further used by producers, manufacturers, and researchers. Several soil moisture models can be fused with the devices for large data generation.

\section{References}

[1] A. Kandwal, A. K. Shakya, R. Prakash, and A. Vidyarthi, "Development of an algorithm for soil moisture retrieval using PALSAR data for bare soil," 2017 International Conference on Information, Communication, Instrumentation and Control (ICICIC), Indore, 2017, pp. 1-6.

[2] J. P. Walker, G. R. Willgoose, and J. D. Kalma, "In situ measurement of soil moisture: a comparison of techniques,” Journal of Hydrology 293 '04, Callaghan, NSW 2308, Australia, pp. 85-99, 2004.

[3] G.J. Gaskin and J.D. Miller, "Measurement of soil water content using a simplified impedance measuring technique", Journal of Agricultural Engineering Research 63, pp. 153-160, 1996.

[4] Rothe, A., Weis, W., Kreutzer, K., Matthies, D., Hess, U., Ansorge, B., "Changes in soil structure caused by the installation of time-domain reflectometry probes and their influence on the measurement of soil moisture". Water Resources. Res. 33 (7), pp 1585-1593, 1997.

[5] Topp, G.C. "State of the art of measuring soil water content," Hydrological Process. 17, pp. 2993-2996, 2003.

[6] Cosh, M.H., Jackson, T.J., Bindlish, R., Famiglietti, J.S., and Ryu, D., "Calibration of an impedance probe for estimation of surface soil water content over large regions", Journal of Hydrology, 311:pp. 49-58. 2005.

[7] O. Merlin J. Walker R. Panciera R. Young J. Kalma E. Kim "Soil moisture measurement in heterogeneous terrain", Proc. MODS IM International Congress Modelling Simulation Modelling Simulation Society, Australia pp. 2604-2610, 2007.

[8] Harmandeep Sharma; Manoj K.Shukla; Paul W.Bosl; Robert Steiner, "Soil moisture sensor calibration, actual evapotranspiration, and crop coefficients for drip-irrigated greenhouse chile peppers, Agricultural Water Management, Volume 179, Pages 81-91, 2017.

[9] González-Teruel, J.D.; Torres-Sánchez, R.; Blaya-Ros, P.J.; Toledo-Moreo, A.B.; Jiménez-Buendía, M.; Soto-Valles, F. Design, and Calibration of a Low-Cost SDI-12 Soil Moisture Sensor. Sensors 2019, 19, 491.

[10] Gao, Z.; Zhu, Y.; Liu, C.; Qian, H.; Cao, W.; Ni, J. Design and Test of a Soil Profile Moisture Sensor Based on Sensitive Soil Layers. Sensors 2018, 18, 1648. 